\title{
Supporting Information: Quantum Confinement Effects on Solvatochromic Shifts of Molecular Solutes
}

\author{
Tommaso Giovannini, ${ }^{\dagger}$ Matteo Ambrosetti, ${ }^{\ddagger}$ and Chiara Cappelli*, ${ }^{*}$ \\ $\dagger$ Department of Chemistry, Norwegian University of Science and Technology, 7491 \\ Trondheim, Norway \\ $\ddagger$ Scuola Normale Superiore, Piazza dei Cavalieri 7, 56126 Pisa, Italy. \\ E-mail: chiara.cappelli@sns.it
}




\section{S1 Computational Protocol and Computational De- tails}

The following computational protocol was exploited: ${ }^{1-4}$

1. Definition of the system: The solute (acrolein, pyridine and pyrimidine) was put at the center of a cubic box containing a number of water molecules large enough to account for solute-solvent interactions. Acrolein, pyridine and pyrimidine geometries were optimized at the B3LYP/aug-cc-pVTZ (acrolein) and B3LYP/aug-cc-pVDZ (pyridine and pyrimidine) level of theory, and RESP (pyridine and pyrimidine) or ChelpG (acrolein) charges were calculated by including solvent effects by means of the PCM approach. ${ }^{5}$

2. Classical Molecular Dynamics (MD) runs: For each system, MD simulations were performed by imposing periodic boundary conditions (PBC) on the cubic box. Each MD run was preceded by an equilibration step. From each MD run, a set of 100 uncorrelated snapshots was extracted.

3. Definition of the the two-layer scheme: For each snapshot, a solute-centered sphere was cut. The radius of the sphere was chosen so to describe all specific water-solute interactions.

4. $Q M / M M$ calculations: $\mathrm{QM} / \mathrm{FQ}_{(\mathrm{CT})}, \mathrm{QM} / \mathrm{FQF} \mu_{(\mathrm{CT})}$ and $\mathrm{QM} / \mathrm{QM}_{\mathrm{w}} / \mathrm{FQ}(\mathrm{F} \mu)$ vertical excitation energies were calculated on the spherical frames obtained at the previous step. The results obtained for each frame were convoluted with a gaussian band-shape and averaged to produce the final spectrum.

Molecular structures were optimized at the B3LYP/aug-cc-pVTZ (acrolein) and B3LYP/augcc-pVDZ level (pyridine and pyrimidine), by including solvent effects by means of the implicit PCM. ${ }^{5}$ MD simulations were performed using GROMACS, ${ }^{6}$ by exploiting $\mathrm{GAFF}^{7}$ in case

of pyridine and pyrimidine and OPLS-AA ${ }^{8}$ in case of acrolein, to describe both intra- and 
inter-molecular interactions. RESP charges were used to account for electrostatic interactions in case of pyridine and pyrimidine, whereas ChelpG ${ }^{9}$ scheme was used to fit the atomic acrolein charges. During the fitting process of ChelpG charges to the electrostatic potential, a constrain was applyed to reproduce acrolein molecular dipole moment. Acrolein-water Van der Waals interactions were treated by relying on the parameterization proposed by Georg et al. ${ }^{10}$ A single molecule of the different solutes was dissolved in a cubic box containing 5226, 5342 and 5324 water molecules in case of acrolein, pyridine and pyrimidine, respectively. TIP3P Force Field was used to describe water molecules. ${ }^{11}$ Pyridine and pyrimidine structures were kept fixed during all the steps of the MD run, whereas acrolein was left free to move by only constraining the fast degrees of freedom, i.e. hydrogen oscillations. The different solutes were brought to $0 \mathrm{~K}$ with the steepest descent minimization procedure and then heated to $298.15 \mathrm{~K}$ in an NVT ensemble using the velocity-rescaling ${ }^{12}$ method with an integration time step of 1.0 fs and a coupling constant of 0.1 ps for 100 ps. NPT simulations (using the Parrinello-Rahman barostat and a coupling constant of $1.0 \mathrm{ps}$ ) for 1 ns were performed to obtain an uniform distribution of molecules in the box. 10 ns production runs in the NVT ensemble were finally carried out, fixing the fastest internal degrees of freedom, i.e. hydrogen atoms, by means of the LINCS algorithm $(\delta \mathrm{t}=2.0 \mathrm{fs})^{13}$ in the case of acrolein. Electrostatic interactions were treated by using particle-mesh Ewald (PME) ${ }^{14}$ method with a grid spacing of $1.2 \AA$ and a spline interpolation of order 4. Intramolecular interactions between atom pairs separated up to three bonds were excluded. The analysis of pyridine and pyrimidine trajectories can be found elsewhere. ${ }^{15}$ A snapshot every 100 ps was extracted in order to obtain a total of 100 uncorrelated snapshots for each system.

For each snapshot a solute-centered sphere with a radius of $15 \AA$ was cut (containing approximately 400 water molecules). On each droplet QM/FQ and QM/FQF $\mu$ vertical excitation energies were calculated by exploiting both LR and cLR regimes. In case of QM/FQ, water molecules were modeled by exploiting polarizable FQ SPC parametrization proposed by 
Giovannini et al. ${ }^{4}$, whereas in case of $\mathrm{QM} / \mathrm{FQF} \mu$ calculations the parametrization proposed by Giovannini et al. ${ }^{16}$ was adopted. QM/MM repulsion parameters were taken from Ref. 17. For the sake of comparison, on the same snapshots additional $Q M / \mathrm{QM}_{\mathrm{w}} / \mathrm{MM}$ calculations, i.e. by including the water molecules which are placed at a distance lower than $3.5 \AA$ with respect to each $\mathrm{QM}$ atom $(17,18$ and 18 water molecules on average for acrolein, pyridine and pyrimidine, respectively), were carried out. Acrolein was described at the CAM-B3LYP/aug-cc-pVDZ level of theory in agreement with previous studies. ${ }^{18}$ Pyridine and Pyrimidine were instead treated at the M06/6-311+G(2df,2p) level, according to Ref. 19. In addition, $\mathrm{QM} / \mathrm{PCM}$ vertical excitation energies were also computed. A cluster approach, in which one (pyridine) or two (acrolein and pyrimidine) water molecules were treated at the QM level, was also considered (named $\mathrm{QM}-\mathrm{QM}_{\mathrm{w}}$ ) Such structures are graphically depicted in Fig. S1. Notice that, according to previous studies ${ }^{20,21}$ the so-called corrected-Linear Response (cLR) regime, ${ }^{15,20}$ i.e. a first order State-Specific correction able to take into account for the relaxation of the QM transition density, was adopted in case of computed QM/PCM, $\mathrm{QM} / \mathrm{FQ}\left({ }_{\mathrm{CT}}\right)$ and $\mathrm{QM} / \mathrm{FQF} \mu\left(_{\mathrm{CT}}\right)$ values of the the $n \rightarrow \pi^{*}$. Linear Response (LR) regime was instead adopted for the $\pi \rightarrow \pi^{*}$ transition. Further details on the implementation of such correction for $\mathrm{QM} / \mathrm{FQ}\left({ }_{\mathrm{CT}}\right)$ and $\mathrm{QM} / \mathrm{FQF} \mu\left(_{\mathrm{CT}}\right)$ can be found in Ref. 15. All QM and QM/MM calculations were performed by using a locally modified version of the Gaussian 16 package. ${ }^{22}$

a.

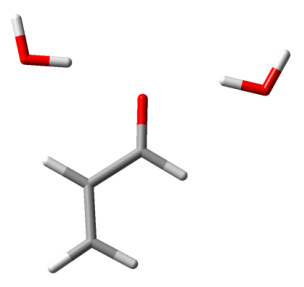

b.

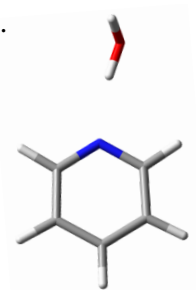

c.

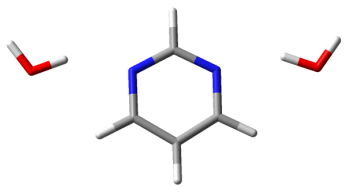

Figure S1: QM-QMw acrolein (a), pyridine (b) and pyrimidine (c) optimized structures. 


\section{S2 Acrolein in aqueous solution}

\section{S2.1 MD Analysis}

In this section, MD runs for acrolein in aqueous solution are analyzed by using the TRAVIS package, ${ }^{23}$ so to extract hydration patterns and analyze the role of hydrogen bonding (HB) interactions. In particular, the radial distribution function $g(r)$ between the solute oxygen atom and solvent hydrogen atoms (HW) are studied, together with the dihedral distribution function (ddf).

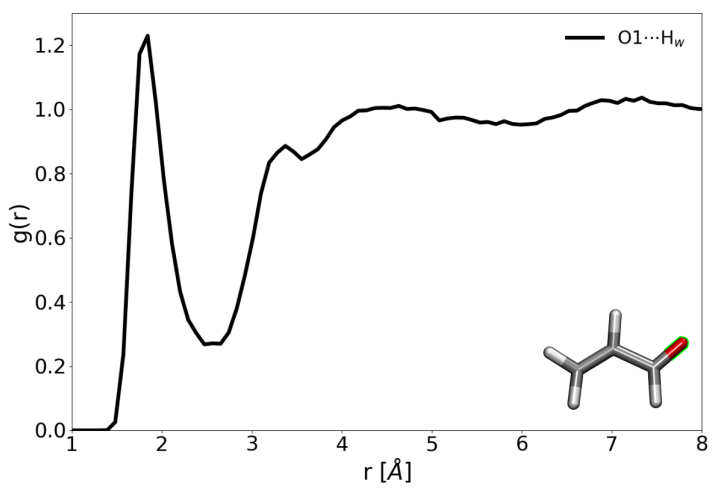

Figure S2: Acrolein radial distribution function of solute oxygen atom with water hydrogen atoms $(\mathrm{HW})$.

$g(r)$ and ddf are reported in Figs. S2 and S3. As it can be evinced, the $g(r)$ presents a sharp peak related to the first solvation shell at about $2 \AA$, thus highlighting the presence of a strong HB interaction. The analysis on ddf instead shows that the main dihedral angle remains almost fixed during MD simulation, with only small fluctuations with respect to the equilibrium value (0 degrees). This is chemically consistent because the two carbon atoms are connected by a planar double covalent bond. 


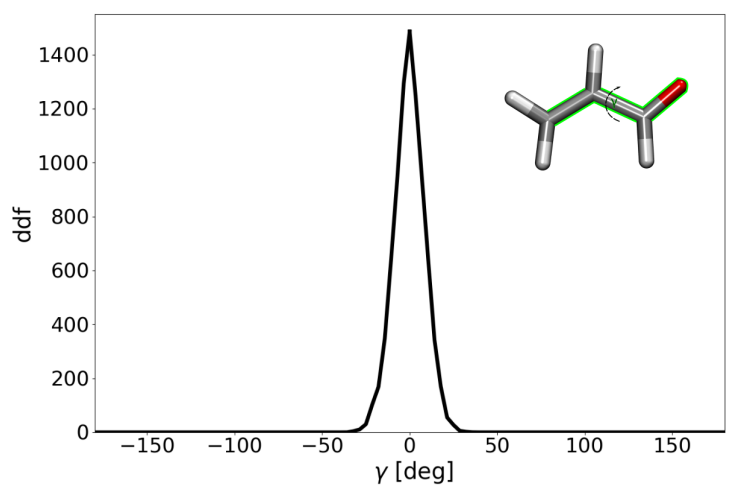

Figure S3: Acrolein dihedral distribution function (ddf) of the highlighted dihedral angle depicted in the inset. 


\section{S2.2 Dependence of the Excitation Energies on the size of the MM-CT region}

Table S1: Acrolein CAM-B3LYP/aug-cc-pVDZ vertical excitation energies as a function of the MM-CT region size, i.e. the $\mathrm{CT}$ is allowed between the water molecules that are placed at a distance lower than 3.5, 5.0, 6.5 and $8.0 \AA$ with respect to each QM atom.

\begin{tabular}{llcccc}
\hline & Model & $3.5 \AA$ & $5.0 \AA$ & $6.5 \AA$ & $8.0 \AA$ \\
\hline \multirow{5}{*}{$n \rightarrow \pi^{*}(\mathrm{cLR})$} & $\mathrm{QM} / \mathrm{FQ}_{\mathrm{CT}}$ & 4.09 & 4.09 & 4.08 & 4.08 \\
& $\mathrm{QM} / \mathrm{FQ}_{\mathrm{CT}}+\mathrm{rep}$ & 4.02 & 4.03 & 4.02 & 4.02 \\
& $\mathrm{QM} / \mathrm{FQF} \mu_{\mathrm{CT}}$ & 4.13 & 4.13 & 4.12 & 4.12 \\
& $\mathrm{QM} / \mathrm{FQF} \mu_{\mathrm{CT}}+\mathrm{rep}$ & 4.04 & 4.05 & 4.05 & 4.05 \\
\hline \multirow{5}{*}{$\pi \rightarrow \pi^{*}(\mathrm{LR})$} & $\mathrm{QM} / \mathrm{FQ} Q_{\mathrm{CT}}$ & 5.67 & 5.68 & 5.68 & 5.68 \\
& $\mathrm{QM} / \mathrm{FQ}_{\mathrm{CT}}+$ rep & 5.88 & 5.89 & 5.88 & 5.88 \\
& $\mathrm{QM} / \mathrm{FQF} \mu_{\mathrm{CT}}$ & 5.62 & 5.60 & 5.60 & 5.60 \\
& $\mathrm{QM} / \mathrm{FQF} \mu_{\mathrm{CT}}+$ rep & 5.80 & 5.81 & 5.79 & 5.79 \\
\hline
\end{tabular}




\section{S2.3 Vertical Excitation Energies}

Table S2: Acrolein CAM-B3LYP/aug-cc-pVDZ vertical excitation energies $\left(\mathrm{E}_{\text {vert }}\right)$ and vacuoto-water solvatochromism $\left(\Delta E_{v a c}^{a q}\right)$. Reference vacuo vertical excitation energies: $n \rightarrow \pi^{*} 3.78$ $\mathrm{eV}\left(\exp ^{18} 3.69 \mathrm{eV}\right) ; \pi \rightarrow \pi^{*} 6.40 \mathrm{eV}\left(\exp ^{18}{ }^{18} 6.42 \mathrm{eV}\right)$. The percentages in $\Delta E_{\text {vac }}^{a q}$ column report the errors with respect to the experimental solvatochromic shift. Percentages in $\Delta E_{\text {rep }}$ column represent the contributions of Pauli repulsion. Percentages in $\Delta E_{\mathrm{CT}}$ column represent the contributions of including intermolecular CT between MM solvent molecules with respect to the basic $\mathrm{QM} / \mathrm{FQ}$ and $\mathrm{QM} / \mathrm{FQF} \mu$ models.

\begin{tabular}{|c|c|c|c|c|c|}
\hline Transition & Method & $\mathrm{E}_{\text {vert }}$ & $\Delta E_{\text {vac }}^{a q}$ & $\Delta E_{\text {rep }}$ & $\Delta E_{\mathrm{CT}}$ \\
\hline \multirow{15}{*}{$n \rightarrow \pi^{*}$} & $\mathrm{QM} / \mathrm{PCM}$ & 3.89 & $-0.11(56 \%)$ & \multirow{4}{*}{$-0.04(17 \%)$} & \multirow{8}{*}{$\begin{array}{l}-0.05(13 \%) \\
-0.05(17 \%)\end{array}$} \\
\hline & QM-QM ${ }_{\mathrm{w}}$ & 4.08 & $-0.30(20 \%)$ & & \\
\hline & QM/TIP3P & 4.02 & $-0.24(4 \%)$ & & \\
\hline & $\mathrm{QM} / \mathrm{TIP} 3 \mathrm{P}+\mathrm{rep}$ & 3.98 & $-0.20(20 \%)$ & & \\
\hline & $\mathrm{QM} / \mathrm{FQ}$ & 4.14 & $-0.36(42 \%)$ & \multirow{2}{*}{$-0.06(16 \%)$} & \\
\hline & $\mathrm{QM} / \mathrm{FQ}+\mathrm{rep}$ & 4.08 & $-0.30(20 \%)$ & & \\
\hline & $\mathrm{QM} / \mathrm{FQ}_{C T}$ & 4.09 & $-0.31(24 \%)$ & \multirow{2}{*}{$-0.06(19 \%)$} & \\
\hline & $\mathrm{QM} / \mathrm{FQ}_{C T}+\mathrm{rep}$ & 4.03 & $-0.25(0 \%)$ & & \\
\hline & $\mathrm{QM} / \mathrm{FQF} \mu$ & 4.09 & $-0.31(24 \%)$ & \multirow{2}{*}{$-0.06(19 \%)$} & \\
\hline & $\mathrm{QM} / \mathrm{FQF} \mu+\mathrm{rep}$ & 4.03 & $-0.25(0 \%)$ & & \\
\hline & $\mathrm{QM} / \mathrm{FQF} \mu_{C T}$ & 4.13 & $-0.35(40 \%)$ & \multirow{2}{*}{$-0.08(23 \%)$} & $+0.04(13 \%)$ \\
\hline & $\mathrm{QM} / \mathrm{FQF} \mu_{C T}+\mathrm{rep}$ & 4.05 & $-0.27(8 \%)$ & & $+0.02(8 \%)$ \\
\hline & $\mathrm{QM} / \mathrm{QM}_{w} / \mathrm{FQ}$ & 4.05 & $-0.27(8 \%)$ & & \\
\hline & $\mathrm{QM} / \mathrm{QM}_{w} / \mathrm{FQF} \mu$ & 4.06 & $-0.28(12 \%)$ & & \\
\hline & $\operatorname{Exp}^{18}$ & 3.94 & -0.25 & & \\
\hline \multirow{15}{*}{$\pi \rightarrow \pi^{*}$} & $\mathrm{QM} / \mathrm{PCM}$ & 6.15 & $0.25(52 \%)$ & \multirow{4}{*}{$0.12(41 \%)$} & \multirow{8}{*}{$\begin{array}{l}-0.17(31 \%) \\
-0.11(28 \%)\end{array}$} \\
\hline & $\mathrm{QM}-\mathrm{QM}_{\mathrm{w}}$ & 6.14 & $0.26(50 \%)$ & & \\
\hline & QM/TIP3P & 6.11 & $0.29(44 \%)$ & & \\
\hline & QM/TIP3P+rep & 6.23 & $0.17(67 \%)$ & & \\
\hline & $\mathrm{QM} / \mathrm{FQ}$ & 5.84 & $0.52(0 \%)$ & \multirow{2}{*}{$0.16(31 \%)$} & \\
\hline & $\mathrm{QM} / \mathrm{FQ}+\mathrm{rep}$ & 6.00 & $0.40(23 \%)$ & & \\
\hline & $\mathrm{QM} / \mathrm{FQ}_{C T}$ & 5.68 & $0.72(38 \%)$ & \multirow{2}{*}{$0.20(28 \%)$} & \\
\hline & $\mathrm{QM} / \mathrm{FQ}_{C T}+\mathrm{rep}$ & 5.89 & $0.51(2 \%)$ & & \\
\hline & $\mathrm{QM} / \mathrm{FQF} \mu$ & 5.84 & $0.56(9 \%)$ & \multirow{2}{*}{$0.16(28 \%)$} & \\
\hline & $\mathrm{QM} / \mathrm{FQF} \mu+\mathrm{rep}$ & 6.00 & $0.40(22 \%)$ & & \\
\hline & $\mathrm{QM} / \mathrm{FQF} \mu_{C T}$ & 5.60 & $0.80(53 \%)$ & \multirow{2}{*}{$0.21(26 \%)$} & \multirow{2}{*}{$\begin{array}{l}-0.23(41 \%) \\
-0.19(46 \%)\end{array}$} \\
\hline & $\mathrm{QM} / \mathrm{FQF} \mu_{C T}+\mathrm{rep}$ & 5.81 & $0.59(14 \%)$ & & \\
\hline & $\mathrm{QM} / \mathrm{QM}_{w} / \mathrm{FQ}$ & 5.77 & $0.63(21 \%)$ & & \\
\hline & $\mathrm{QM} / \mathrm{QM}_{w} / \mathrm{FQF} \mu$ & 5.80 & $0.60(15 \%)$ & & \\
\hline & $\operatorname{Exp}^{18}$ & 5.90 & 0.52 & & \\
\hline
\end{tabular}


Table S3: Acrolein QM/FQ and QM/FQF $\mu \omega_{0}$, LR and $\operatorname{cLR} n \rightarrow \pi^{*}$ and $\pi \rightarrow \pi^{*}$ vertical excitation energies. The results used for the analysis in the main text are in bold (i.e. cLR and LR excitation energies in case of $n \rightarrow \pi^{*}$ and $\pi \rightarrow \pi^{*}$ transitions, respectively). The QM portion is described at the CAM-B3LYP/aug-cc-pVDZ level. All data are given in eV.

\begin{tabular}{|c|c|c|c|c|c|c|}
\hline \multirow{2}{*}{ Method } & \multicolumn{3}{|c|}{$n \rightarrow \pi^{*}$} & \multicolumn{3}{|c|}{$\pi \rightarrow \pi^{*}$} \\
\hline & $\omega_{0}$ & LR & cLR & $\omega_{0}$ & LR & $\mathrm{cLR}$ \\
\hline $\mathrm{QM} / \mathrm{PCM}$ & 3.94 & 3.94 & 3.89 & 6.29 & 6.15 & 6.27 \\
\hline $\mathrm{QM} / \mathrm{FQ}$ & 4.16 & 4.15 & 4.14 & 5.97 & 5.84 & 5.94 \\
\hline $\mathrm{QM} / \mathrm{FQ}+\mathrm{rep}$ & 4.11 & 4.10 & 4.08 & 6.11 & 6.00 & 6.09 \\
\hline $\mathrm{QM} / \mathrm{FQ}_{C T}$ & 4.14 & 4.14 & 4.09 & 5.98 & 5.68 & 5.91 \\
\hline $\mathrm{QM} / \mathrm{FQ}_{C T}+\mathrm{rep}$ & 4.09 & 4.08 & 4.03 & 6.12 & 5.89 & 6.07 \\
\hline & 4.12 & 4.12 & 4.09 & 6.01 & 5.84 & 5.97 \\
\hline $\mathrm{QM} /$ & 4.07 & 4.07 & 4.03 & 6.14 & 6.00 & 6.11 \\
\hline $\mathrm{QM} /$ & 4.18 & 4.18 & 4.13 & 5.95 & 5.60 & 5.87 \\
\hline $\mathrm{QM} / \mathrm{FQF} \mu_{C T}+\mathrm{rep}$ & 4.12 & 4.12 & 4.05 & 6.10 & 5.81 & 6.03 \\
\hline
\end{tabular}




\section{S3 Pyridine in aqueous solution}

Table S4: Pyridine QM/FQ and QM/FQF $\mu \omega_{0}$, LR and cLR $n \rightarrow \pi^{*}$ vertical excitation energies. The results used for the analysis in the main text are in bold (cLR). The QM portion is described at the M06/6-311+G(2df,2p) level. All data are given in $\mathrm{eV}$.

\begin{tabular}{l|ccc}
\hline \multirow{2}{*}{ Method } & \multicolumn{3}{|c}{$n \rightarrow \pi^{*}$} \\
& $\omega_{0}$ & LR & cLR \\
\hline QM/PCM & 4.84 & 4.84 & 4.79 \\
QM/FQ & 5.30 & 5.28 & 5.18 \\
QM/FQ+rep & 5.07 & 5.06 & 5.03 \\
QM/FQ $C T$ & 5.22 & 5.20 & 5.07 \\
QM/FQ $C T+$ rep & 5.08 & 5.06 & 4.97 \\
QM/FQF $\mu$ & 5.21 & 5.20 & 5.12 \\
QM/FQF $\mu+$ rep & 5.06 & 5.05 & 5.00 \\
QM/FQF $\mu_{C T}$ & 5.27 & 5.24 & 5.11 \\
QM/FQF $\mu_{C T}+$ rep & 5.12 & 5.10 & 5.01 \\
\hline
\end{tabular}


Table S5: Pyridine M06/6-311+G(2df,2p) vertical excitations and vacuo-to-water solvatochromism. Reference vacuo vertical excitation energy: $4.67 \mathrm{eV}$ (exp. ${ }^{19} 4.59,4.63$ and 4.74 $\mathrm{eV})$. The percentages in $\Delta E_{v a c}^{a q}$ column represent errors with respect to the experimental solvatochromic shift. Percentages in $\Delta E_{\text {rep }}$ column represent the contributions of including Pauli repulsion. Percentages in $\Delta E_{\mathrm{CT}}$ column are the contribution of intermolecular CT between MM solvent molecules with respect to the basic QM/FQ and QM/FQF $\mu$ models.

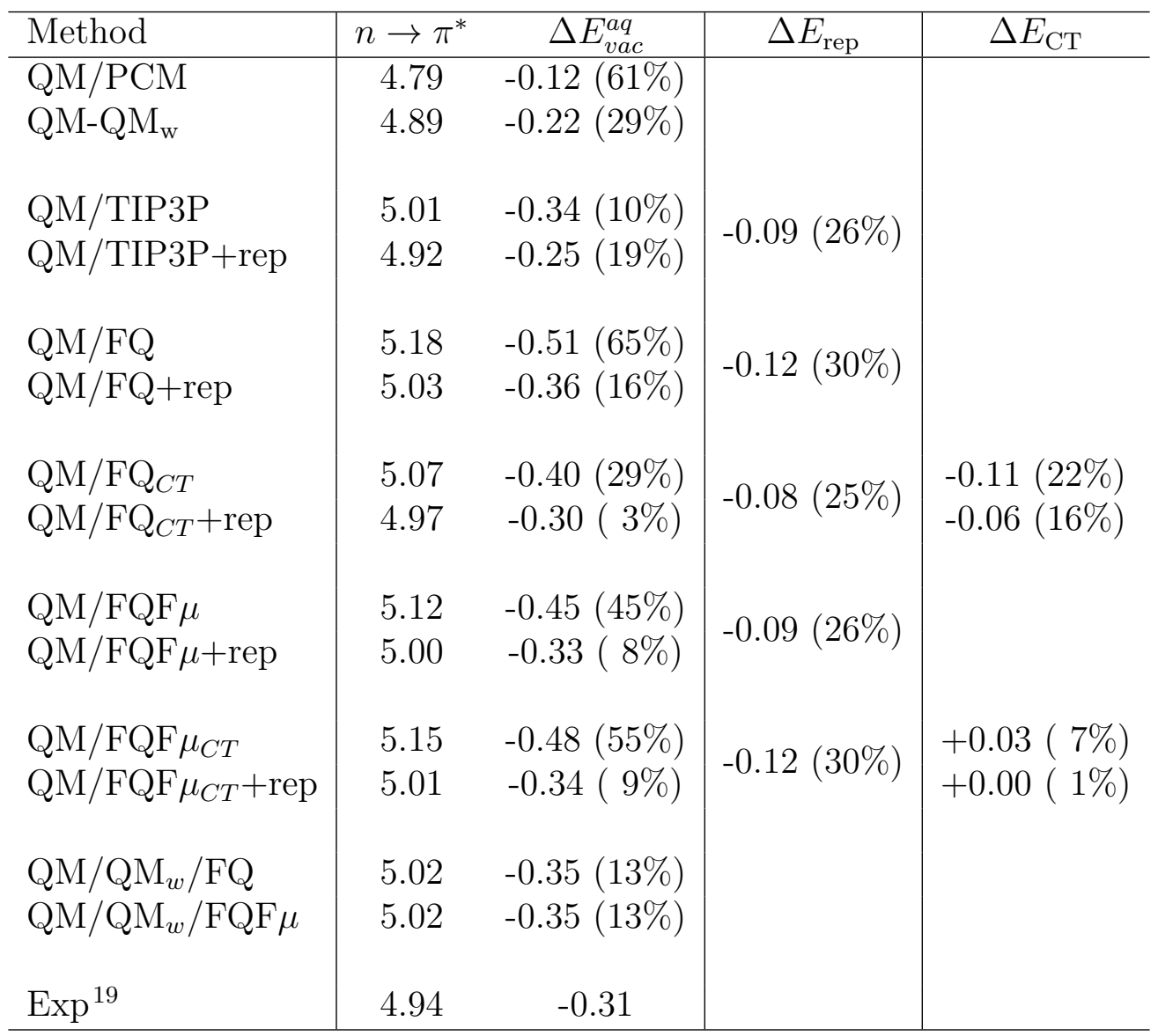




\section{S4 Pyrimidine in aqueous solution}

Table S6: Pyrimidine QM/FQ and QM/FQF $\mu \omega_{0}$, LR and cLR $n \rightarrow \pi^{*}$ vertical excitation energies. The results used for the analysis in the main text are in bold (cLR). The QM portion is described at the M06/6-311+G(2df,2p) level. All data are given in $\mathrm{eV}$.

\begin{tabular}{l|ccc}
\hline \multirow{2}{*}{ Method } & \multicolumn{3}{|c}{$n \rightarrow \pi^{*}$} \\
& $\omega_{0}$ & LR & cLR \\
\hline QM/PCM & 4.31 & 4.30 & 4.28 \\
QM/FQ & 4.69 & 4.69 & 4.67 \\
QM/FQ+rep & 4.52 & 4.52 & 4.50 \\
QM/FQ $C T$ & 4.70 & 4.68 & 4.64 \\
QM/FQ $C T+$ rep & 4.52 & 4.51 & 4.47 \\
$\mathrm{QM} / \mathrm{FQF} \mu$ & 4.67 & 4.66 & 4.63 \\
$\mathrm{QM} / \mathrm{FQF} \mu+\mathrm{rep}$ & 4.45 & 4.45 & 4.43 \\
$\mathrm{QM} / \mathrm{FQF} \mu_{C T}$ & 4.72 & 4.71 & 4.65 \\
$\mathrm{QM} / \mathrm{FQF} \mu_{C T}+\mathrm{rep}$ & 4.53 & 4.52 & 4.47 \\
\hline
\end{tabular}


Table S7: Pyrimidine M06/6-311+G(2df,2p) vertical excitations and vacuo-to-water solvatochromism. Reference vacuo vertical excitation energy: $4.17 \mathrm{eV}$ (exp. ${ }^{19} 4.18 \pm 0.01 \mathrm{eV}$ ). Percentages in $\Delta E_{v a c}^{a q}$ column give errors with respect to the experimental solvatochromic shift. Percentages in $\Delta E_{\text {rep }}$ column represent the contributions of including Pauli repulsion. Percentages in $\Delta E_{\mathrm{CT}}$ column represent the contribution of including intermolecular CT between MM solvent molecules with respect to the basic QM/FQ and QM/FQF $\mu$ models.

\begin{tabular}{|c|c|c|c|c|}
\hline Method & $n \rightarrow \pi^{*}$ & $\Delta E_{v a c}^{a q}$ & $\Delta E_{\mathrm{rep}}$ & $\Delta E_{\mathrm{CT}}$ \\
\hline $\mathrm{QM} / \mathrm{PCM}$ & 4.28 & $-0.11(69 \%)$ & \multirow{4}{*}{$-0.16(47 \%)$} & \multirow{15}{*}{$\begin{array}{l}-0.03(6 \%) \\
-0.03(9 \%)\end{array}$} \\
\hline $\mathrm{QM}^{-} \mathrm{QM}_{\mathrm{w}}$ & 4.43 & $-0.26(26 \%)$ & & \\
\hline QM/TIP3P & 4.51 & $-0.34(3 \%)$ & & \\
\hline QM/TIP3P+rep & 4.35 & $-0.18(49 \%)$ & & \\
\hline $\mathrm{QM} / \mathrm{FQ}$ & 4.67 & $-0.50(43 \%)$ & \multirow{2}{*}{$-0.17(34 \%)$} & \\
\hline $\mathrm{QM} / \mathrm{FQ}+\mathrm{rep}$ & 4.50 & $-0.33(6 \%)$ & & \\
\hline $\mathrm{QM} / \mathrm{FQ}_{C T}$ & 4.64 & $-0.47(34 \%)$ & \multirow{2}{*}{$-0.17(36 \%)$} & \\
\hline $\mathrm{QM} / \mathrm{FQ}_{C T}+\mathrm{rep}$ & 4.47 & $-0.30(14 \%)$ & & \\
\hline $\mathrm{QM} / \mathrm{FQF} \mu$ & 4.63 & $-0.46(31 \%)$ & \multirow{2}{*}{$-0.20(43 \%)$} & \\
\hline $\mathrm{QM} / \mathrm{FQF} \mu+\mathrm{rep}$ & 4.43 & $-0.26(26 \%)$ & & \\
\hline $\mathrm{QM} / \mathrm{FQF} \mu_{C T}$ & 4.65 & $-0.48(37 \%)$ & \multirow{5}{*}{$-0.18(38 \%)$} & \\
\hline $\mathrm{QM} / \mathrm{FQF} \mu_{C T}+\mathrm{rep}$ & 4.47 & $-0.30(14 \%)$ & & \\
\hline $\mathrm{QM} / \mathrm{QM}_{w} / \mathrm{FQ}$ & 4.48 & $-0.31(11 \%)$ & & \\
\hline $\mathrm{QM} / \mathrm{QM}_{w} / \mathrm{FQF} \mu$ & 4.49 & $-0.32(9 \%)$ & & \\
\hline $\operatorname{Exp}^{19}$ & 4.57 & $-0.35 \pm 0.06$ & & \\
\hline
\end{tabular}




\section{S5 Molecular Dipole Moments}

Table S8: Acrolein, pyridine and pyrimidine computed Ground State (gs) and Excited State $\left(n \rightarrow \pi^{*}\right.$ and $\left.\pi \rightarrow \pi^{*}\right)$ electric dipole moments. All data are given in Debye.

\begin{tabular}{l|lll|ll|ll}
\hline \multirow{2}{*}{ Method } & \multicolumn{3}{|c|}{ acrolein } & \multicolumn{3}{c}{ pyridine } & \multicolumn{2}{c}{ pyrimidine } \\
& gs & $n \rightarrow \pi^{*}$ & $\pi \rightarrow \pi^{*}$ & gs & $n \rightarrow \pi^{*}$ & gs & $n \rightarrow \pi^{*}$ \\
\hline Vacuo & 3.5 & 1.0 & 4.5 & 2.3 & 0.4 & 2.4 & 0.6 \\
\hline QM/FQ & 5.8 & 3.4 & 7.8 & 4.4 & 1.7 & 4.4 & 2.8 \\
QM/FQ+rep & $5.3(9 \%)$ & $3.0(12 \%)$ & $7.4(5 \%)$ & $3.8(14 \%)$ & $1.4(18 \%)$ & $3.7(15 \%)$ & $2.2(21 \%)$ \\
\hline QM/FQ $C T$ & 5.9 & 3.1 & 8.2 & 4.5 & 1.3 & 4.4 & 2.8 \\
QM/FQ $C T+$ rep & $5.3(10 \%)$ & $2.7(13 \%)$ & $7.6(7 \%)$ & $3.7(18 \%)$ & $0.9(31 \%)$ & $3.7(16 \%)$ & $1.9(32 \%)$ \\
\hline QM/FQF $\mu$ & 5.6 & 3.1 & 7.7 & 4.4 & 1.6 & 4.4 & 2.7 \\
QM/FQF $\mu+$ rep & $5.2(7 \%)$ & $2.7(13 \%)$ & $7.3(5 \%)$ & $3.8(14 \%)$ & $1.3(19 \%)$ & $3.4(23 \%)$ & $1.7(37 \%)$ \\
\hline QM/FQF $\mu_{C T}$ & 6.2 & 3.3 & 8.6 & 5.0 & 1.6 & 4.7 & 3.0 \\
QM/FQF $\mu_{C T}+$ rep & $5.5(11 \%)$ & $2.8(15 \%)$ & $7.8(9 \%)$ & $4.1(18 \%)$ & $1.2(25 \%)$ & $4.0(15 \%)$ & $2.1(30 \%)$ \\
\hline
\end{tabular}

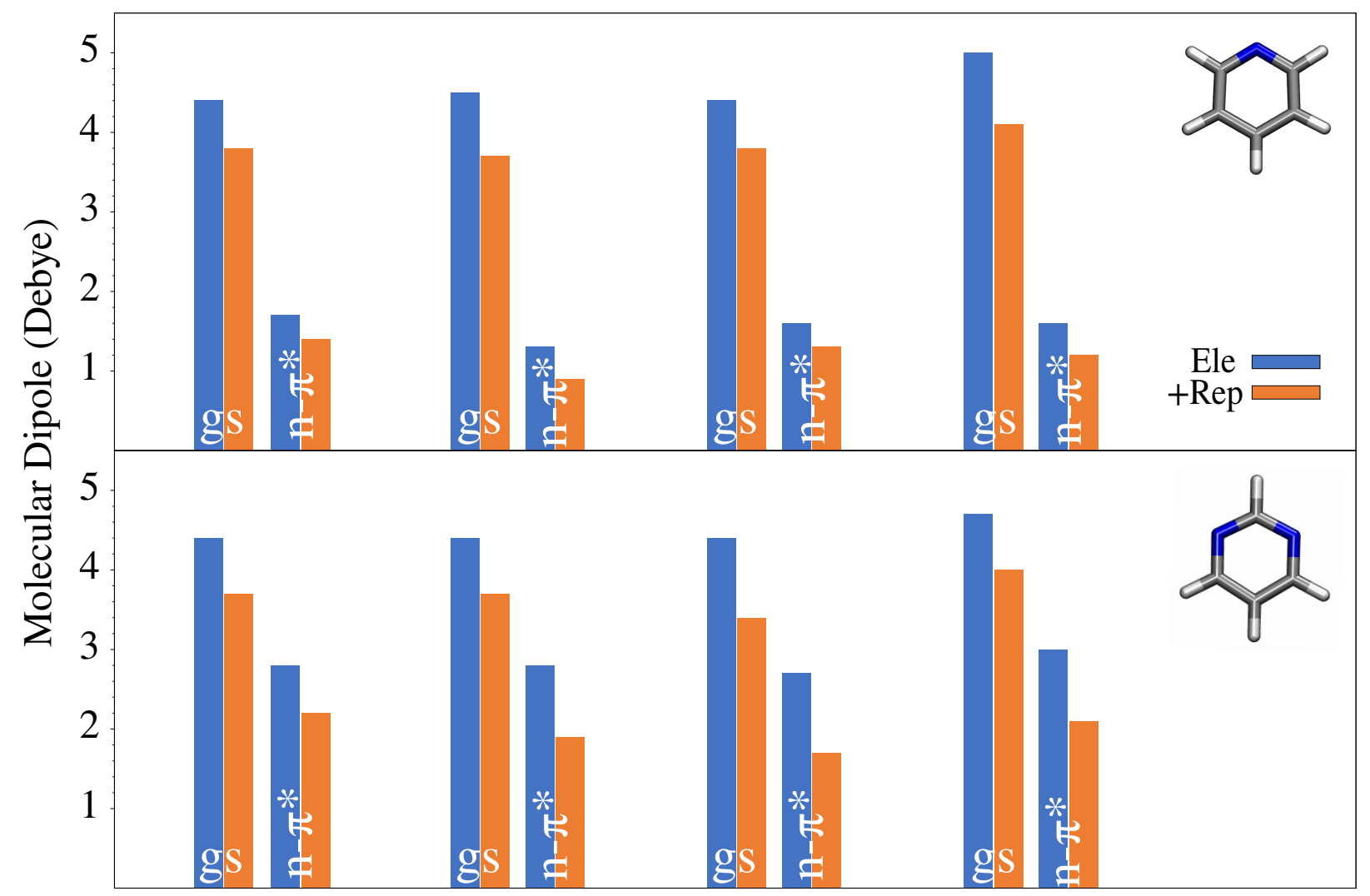

Figure S4: Computed QM/FQ, QM/FQ ${ }_{\mathrm{CT}}, \mathrm{QM} / \mathrm{FQF} \mu$ and $\mathrm{QM} / \mathrm{FQF} \mu_{\mathrm{CT}}$ pyridine (top) and pyrimidine (bottom) ground and excited state molecular dipole moments. Data are given in Table S8. All data are in Debye. 


\section{References}

(1) Giovannini, T.; Del Frate, G.; Lafiosca, P.; Cappelli, C. Effective computational route towards vibrational optical activity spectra of chiral molecules in aqueous solution. Phys. Chem. Chem. Phys. 2018, 20, 9181-9197.

(2) Giovannini, T.; Macchiagodena, M.; Ambrosetti, M.; Puglisi, A.; Lafiosca, P.; Lo Gerfo, G.; Egidi, F.; Cappelli, C. Simulating vertical excitation energies of solvated dyes: From continuum to polarizable discrete modeling. Int. J. Quantum Chem. 2019, 119, e25684.

(3) Giovannini, T.; Ambrosetti, M.; Cappelli, C. A polarizable embedding approach to second harmonic generation (SHG) of molecular systems in aqueous solutions. Theor. Chem. Acc. 2018, $137,74$.

(4) Giovannini, T.; Lafiosca, P.; Chandramouli, B.; Barone, V.; Cappelli, C. Effective yet Reliable Computation of Hyperfine Coupling Constants in Solution by a QM/MM Approach: Interplay Between Electrostatics and Non-electrostatic Effects. J. Chem. Phys. 2019, 150, 124102.

(5) Tomasi, J.; Mennucci, B.; Cammi, R. Quantum mechanical continuum solvation models. Chem. Rev. 2005, 105, 2999-3094.

(6) Abrahama, M. J.; Murtola, T.; Schulz, R.; Pálla, S.; Smith, J. C.; Hess, B.; Lindahl, E. GROMACS: High Performance Molecular Simulations through Multi-Level Parallelism from Laptops to Supercomputers. SoftwareX 2015, 1-2, 19-25.

(7) Oostenbrink, C.; Villa, A.; Mark, A. E.; Van Gunsteren, W. F. A biomolecular force field based on the free enthalpy of hydration and solvation: The GROMOS force-field parameter sets 53A5 and 53A6. J. Comput. Chem. 2004, 25, 1656-1676.

(8) Jorgensen, W. L.; Maxwell, D. S.; Tirado-Rives, J. Development and testing of the OPLS all-atom force field on conformational energetics and properties of organic liquids. J. Am. Chem. Soc. 1996, 118, 11225-11236. 
(9) Breneman, C. M.; Wiberg, K. B. Determining atom-centered monopoles from molecular electrostatic potentials. The need for high sampling density in formamide conformational analysis. J. Comput. Chem. 1990, 11, 361-373.

(10) Georg, H. C.; Coutinho, K.; Canuto, S. A sequential Monte Carlo quantum mechanics study of the hydrogen-bond interaction and the solvatochromic shift of the $\mathrm{n}-\pi^{*}$ transition of acrolein in water. J. Chem. Phys. 2005, 123, 124307.

(11) Mark, P.; Nilsson, L. Structure and dynamics of the TIP3P, SPC, and SPC/E water models at 298 K. J. Phys. Chem. A 2001, 105, 9954-9960.

(12) Bussi, G.; Donadio, D.; Parrinello, M. Canonical sampling through velocity rescaling. J. Chem. Phys. 2007, 126.

(13) Hess, B.; Bekker, H.; Berendsen, H. J.; Fraaije, J. G. LINCS: a linear constraint solver for molecular simulations. J. Comput. Chem. 1997, 18, 1463-1472.

(14) Darden, T.; York, D.; Pedersen, L. Particle mesh Ewald: An Nlog(N) method for Ewald sums in large systems. J. Chem. Phys. 1993, 98, 10089-10092.

(15) Giovannini, T.; Riso, R. R.; Ambrosetti, M.; Puglisi, A.; Cappelli, C. Electronic Transitions for a Fully Polarizable QM/MM Approach Based on Fluctuating Charges and Fluctuating Dipoles: Linear and Corrected Linear Response Regimes. arXiv e-prints 2019, arXiv:1906.03852.

(16) Giovannini, T.; Puglisi, A.; Ambrosetti, M.; Cappelli, C. Polarizable QM/MM approach with fluctuating charges and fluctuating dipoles: the QM/FQF $\mu$ model. J. Chem. Theory Comput. 2019, 15, 2233-2245.

(17) Giovannini, T.; Lafiosca, P.; Cappelli, C. A General Route to Include Pauli Repulsion and Quantum Dispersion Effects in QM/MM Approaches. J. Chem. Theory Comput. 2017, 13, $4854-4870$.

(18) Aidas, K.; Møgelhøj, A.; Nilsson, E. J.; Johnson, M. S.; Mikkelsen, K. V.; Christiansen, O.; Söderhjelm, P.; Kongsted, J. On the performance of quantum chemical methods to predict 
solvatochromic effects: The case of acrolein in aqueous solution. J. Chem. Phys. 2008, 128, 194503.

(19) Marenich, A. V.; Cramer, C. J.; Truhlar, D. G. Electronic absorption spectra and solvatochromic shifts by the vertical excitation model: solvated clusters and molecular dynamics sampling. J. Phys. Chem. B 2015, 119, 958-967.

(20) Caricato, M.; Mennucci, B.; Tomasi, J.; Ingrosso, F.; Cammi, R.; Corni, S.; Scalmani, G. Formation and relaxation of excited states in solution: A new time dependent polarizable continuum model based on time dependent density functional theory. J. Chem. Phys. 2006, 124, 124520.

(21) Duchemin, I.; Guido, C. A.; Jacquemin, D.; Blase, X. The Bethe-Salpeter formalism with polarisable continuum embedding: reconciling linear-response and state-specific features. Chem. Sci. 2018, 9, 4430-4443.

(22) Frisch, M. J.; Trucks, G. W.; Schlegel, H. B.; Scuseria, G. E.; Robb, M. A.; Cheeseman, J. R.; Scalmani, G.; Barone, V.; Petersson, G. A.; Nakatsuji, H.; Li, X.; Caricato, M.; Marenich, A. V.; Bloino, J.; Janesko, B. G.; Gomperts, R.; Mennucci, B.; Hratchian, H. P.; Ortiz, J. V.; Izmaylov, A. F.; Sonnenberg, J. L.; Williams-Young, D.; Ding, F.; Lipparini, F.; Egidi, F.; Goings, J.; Peng, B.; Petrone, A.; Henderson, T.; Ranasinghe, D.; Zakrzewski, V. G.; Gao, J.; Rega, N.; Zheng, G.; Liang, W.; Hada, M.; Ehara, M.; Toyota, K.; Fukuda, R.; Hasegawa, J.; Ishida, M.; Nakajima, T.; Honda, Y.; Kitao, O.; Nakai, H.; Vreven, T.; Throssell, K.; Montgomery, J. A., Jr.; Peralta, J. E.; Ogliaro, F.; Bearpark, M. J.; Heyd, J. J.; Brothers, E. N.; Kudin, K. N.; Staroverov, V. N.; Keith, T. A.; Kobayashi, R.; Normand, J.; Raghavachari, K.; Rendell, A. P.; Burant, J. C.; Iyengar, S. S.; Tomasi, J.; Cossi, M.; Millam, J. M.; Klene, M.; Adamo, C.; Cammi, R.; Ochterski, J. W.; Martin, R. L.; Morokuma, K.; Farkas, O.; Foresman, J. B.; Fox, D. J. Gaussian 16 Revision A.03. 2016; Gaussian Inc. Wallingford CT.

(23) Brehm, M.; Kirchner, B. TRAVIS - A Free Analyzer and Visualizer for Monte Carlo and Molecular Dynamics Trajectories. J. Chem. Inf. Model. 2011, 51, 2007-2023, PMID: 21761915. 